Supporting Information

\title{
Effective Viscosity of Unentangled Random Copolymer Films of Styrene and 4-Methoxystyrene with Different Copolymer \\ Compositions
}

Jianquan $\mathrm{Xu},{ }^{1}$ Chao Lv, ${ }^{2}$ Binyang Du, ${ }^{2}$ Xinping Wang, ${ }^{3}$ Ophelia K. C. Tsui ${ }^{1, *}$

${ }^{1}$ Department of Physics, Hong Kong University of Science and Technology, Clear Water Bay, Kowloon 999077, Hong Kong, China

${ }^{2}$ MOE Key Laboratory of Macromolecular Synthesis and Functionalization, Department of Polymer Science \& Engineering, Zhejiang University, Hangzhou 310027, China

${ }^{3}$ Department of Chemistry, Zhejiang Sci-Tech University, Hangzhou 310018, China

*Corresponding author:

Email: okctsui@ust.hk 


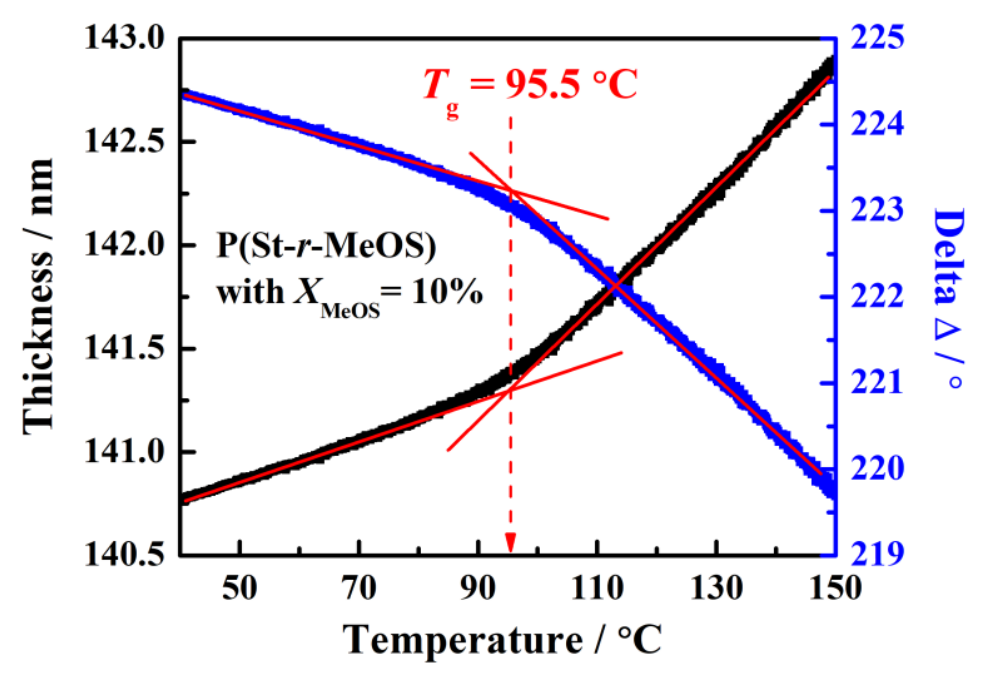

Figure S1. Representative measurements of film thickness, $h_{0}$, and the ellipsometric angle delta, $\Delta$, as a function of temperature, $T$. The value of $T_{\mathrm{g}}$ was determined as the intersection between two straight-line fits to the data (red solid lines) in the glassy and rubber regions.
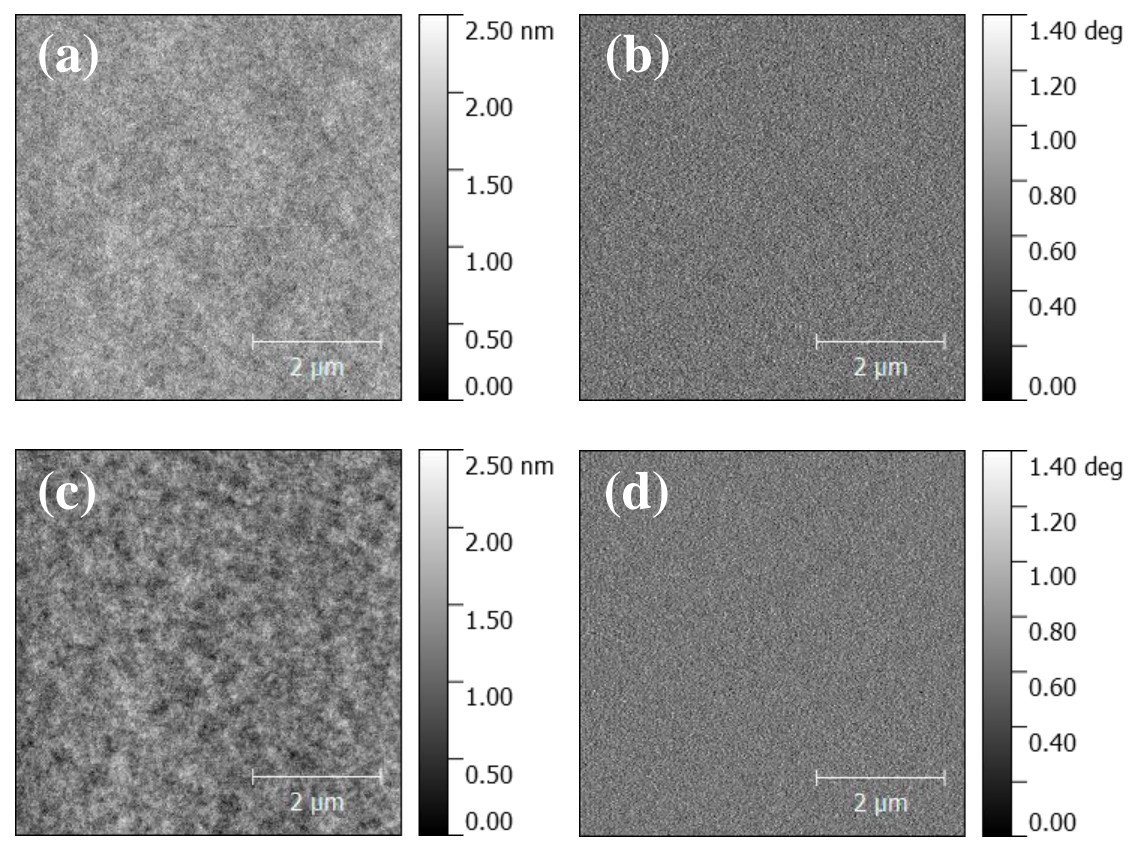

Figure S2. Representatively AFM height and phase images obtained before and after annealing at $130{ }^{\circ} \mathrm{C}$. Height image (a) and phase image (b) of a $14 \mathrm{~nm}$ thick $\mathrm{P}(\mathrm{St}-r$-MeOS) film with $X_{\mathrm{MeOS}}=48 \%$ before annealing. (c) and (d) show the corresponding images of the film after annealing at $130{ }^{\circ} \mathrm{C}$ for $70 \mathrm{~min}$. 


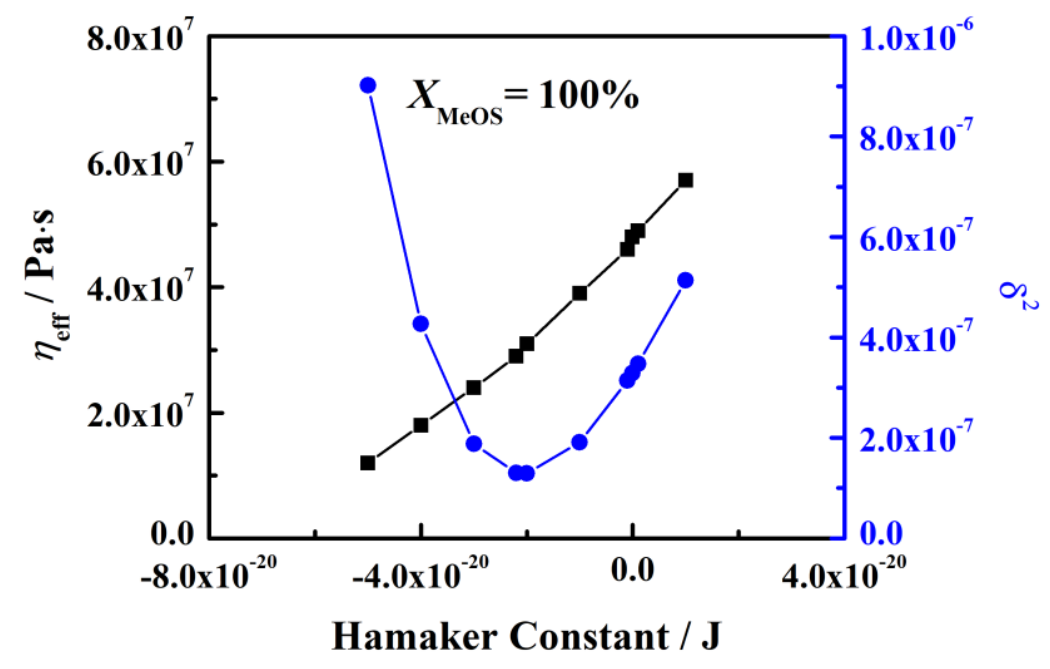

Figure S3. Fit value of $\eta_{\text {eff }}$ (square) and corresponding mean-square percentage difference, $\delta^{2}$, (see eq. S1) (circle) of $3.2 \mathrm{~nm} \mathrm{PMeOS} \mathrm{film}\left(M_{\mathrm{n}}=16.0 \mathrm{~kg} / \mathrm{mol}\right)$ as a function of Hamaker constant employed in fitting the power spectral density data of the film taken at $130^{\circ} \mathrm{C}$ using eqs. $1 \mathrm{a}$ and $1 \mathrm{~b}$.

The parameter, mean-square percentage difference, $\delta^{2}$, plotted in Figure S3 measures the discrepancy between the experimental data and model fit values as defined by.

$$
\delta^{2}=\frac{1}{\left(N_{i}-1\right)\left(N_{j}\right)} \sum_{i=1, j=1}^{N_{i}, N_{j}} \frac{\left[I_{q_{j}}{ }^{2}\left(t_{i}\right)_{\text {theory }}-I_{q_{j}}{ }^{2}\left(t_{i}\right)_{\text {expt }}\right]^{2}}{I_{q_{j}}{ }^{2}\left(t_{i}\right)_{\text {theory }}} .
$$




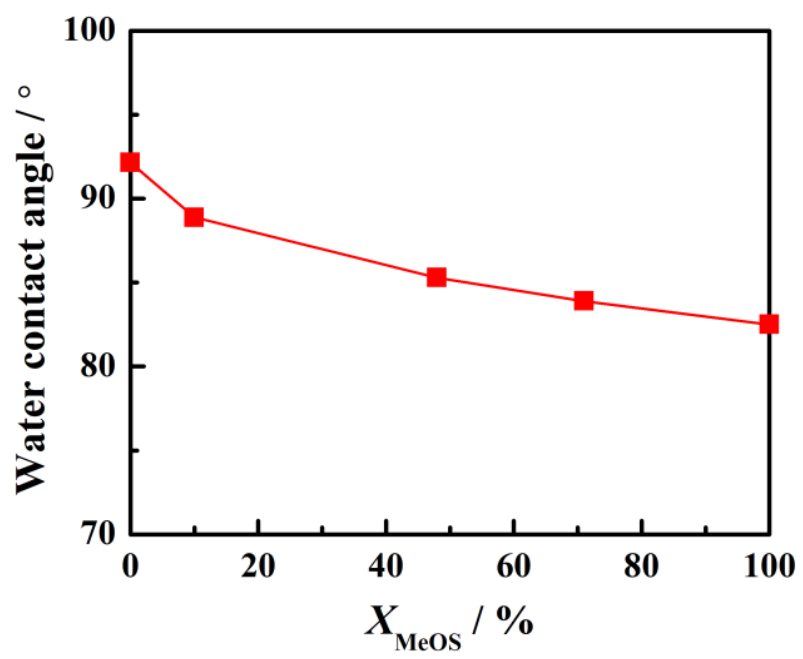

Figure S4. Water contact angle of $\mathrm{P}(\mathrm{St}-r-\mathrm{MeOS})$ films $\left(h_{0} \sim 100 \mathrm{~nm}\right)$ plotted as a function of $X_{\text {MeOs. }}$ Each data point is based on the average value of 6 measurements.

\section{Interfacial Potentials of our P(St-r-MeOS) films}

Table S1 displays fit values of the Hamaker constant, $A$, we found by fitting the $I_{\mathrm{q}}{ }^{2}(t)$ data of the $3 \mathrm{~nm}$ films as illustrated in Figure S3. As one can see, the Hamaker constant of PSt is positive while those of the films containing MeOS are negative. Furthermore, the values swing significantly in going from $X_{\mathrm{MeOS}}=0$ to $48 \%$. As discussed in in ref. 1 and will be briefly discussed below, Hamaker constant parameter $A$ is an overarching parameter summarizing contributions from a variety of components that combine in a nontrivial manner to produce the interfacial potential, $G\left(h_{0}\right)$. Evidently, the large swing in $A$ between $X_{\mathrm{MeOS}}=0$ and $48 \%$ occurs because $A$ changes sign there. (The specific sign change seen here corresponds to the thin film system going from being unstable at $X_{\mathrm{MeOS}}=0$ to being stable at $X_{\mathrm{MeOS}}=48 \%{ }^{1}$ To be able to comprehend the values of $A$ there would require high-precision modeling of the dispersion forces in the system, which is a significant effort ${ }^{1,2}$ and beyond the scope of this work. In the following, we will focus on attaining a qualitative description 
of $G\left(h_{0}\right)$ for the $X_{\mathrm{MeOS}}=48 \%, 71 \%$ and $100 \%$ films in order to gain insight into how the range of $G\left(h_{0}\right)$ varies with $X_{\mathrm{MeOS}}$. We will not estimate $G\left(h_{0}\right)$ for $X_{\mathrm{MeOS}}=0 \%$ and $10 \%$ where $A$ changes sign because we think that simple treatments like the one afforded here could produce erroneous insight.

Table S1. Hamaker constant fit values of the 3nm P(St-r-MeOS) films

\begin{tabular}{|c|c|c|c|c|c|}
\hline$X_{\mathrm{MeOS}}$ & 0 & $10 \%$ & $48 \%$ & $71 \%$ & $100 \%$ \\
\hline$A\left(10^{-21} \mathrm{~J}\right)$ & 8.2 & -11 & -6.5 & -9.3 & -20 \\
\hline
\end{tabular}

The main goal of this calculation is to obtain an estimate for the range of dispersion forces in our films and compare it with the propagation distances of the substrate effect $\left(l_{t}\right)$ displayed in Table 2. In systems devoid of hydrogen bonds, as is the case for our films, intermolecular interactions are dominated by van der Waals dispersion forces. For polymer films supported by oxide-coated silicon (i.e., air/polymer/SiOx/Si, see Figure S5), temporary fluctuating dipoles in the polymer, the $\mathrm{SiO} x$ cover-layer and silicon all contribute to $G\left(h_{0}\right)$. As discussed in the main text, an approximated expression of $G\left(h_{0}\right)$ as given by eq. 3, where retardation effects are neglected, is commonly used. By using eq. 3, and assuming the values of $A$ in Table S1 and $8 \times 10^{-20}$ and $2.34 \times 10^{-19} \mathrm{~J}$ for the values of $A_{2}$ and $A_{3}$, respectively, ${ }^{1}$ we estimated the values of $A_{1}$ (as displayed in Table S2) and thereby $G\left(h_{0}\right)$.

It should be mentioned that the calculation of $G\left(h_{0}\right)$ described above is highly oversimplified. For example, retardation effects were neglected. ${ }^{1}$ Furthermore, there is likely to be densification of polymer chains on the substrate surfaces ${ }^{3}$ with $X_{\mathrm{MeOS}}$ and a change in 
the orientation of the phenyl rings near the substrate (from parallel to perpendicular to the interface). ${ }^{4}$ Negligence of these effects will understandably introduce inaccuracy to the calculation. In fact, we found that in order to fit all the values of $A$ displayed in Table S1 using eqs. $3-5$, we had to assume $A_{2}$ to be larger than the published value of $6.66 \times 10^{-20} \mathrm{~J} .^{1}$ The value of $A_{2}$ that is able to fit the current experiment and the closest to the published value is $8 \times 10^{-20} \mathrm{~J}$. Recall that $A_{2}$ is the Hamaker constant of $\mathrm{SiO} x$. We perceive the larger value of $A_{2}$ required to fit the current data may be caused by densification of polymer chains and alignment of the phenyl rings near the substrate. ${ }^{4}$

Air (0)

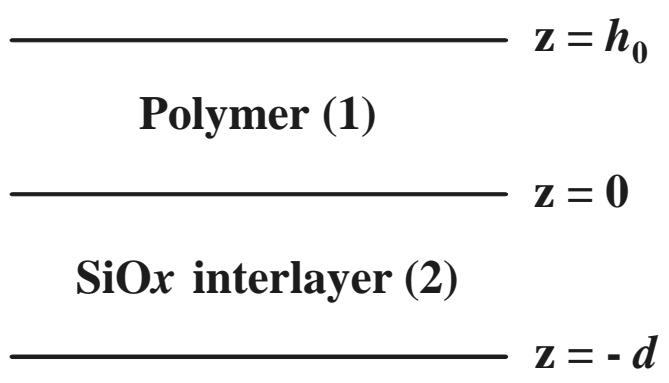

Si substrate (3)

Figure. S5 Schematic of the layer structure of the films and designation of the material number $i$ in eq. 3 .

Table S2 displays the fit values of $A_{1}$. As one can see, $A_{1}$ increases with $X_{\text {MeOs. In PS, }}$ dispersion interactions are promoted by delocalization of electrons in the phenyl rings (Figure 1), which allows larger induced dipole moments than if electron delocalization was absent. With addition of a MeOS group to a phenyl ring (Figure 1), some of the lone-pair electrons in 
the oxygen of the MeOS group may also delocalize into the phenyl ring, ${ }^{5}$ rendering enhancement in the polarizability of the substituted phenyl rings and thereby the Hamaker constant $A_{1}$.

Table S2. Hamaker constants $A_{1}$ of our $\mathrm{P}(\mathrm{St}-\mathrm{r}$-MeOS) polymers calculated as described in the text.

\begin{tabular}{|c|c|c|c|}
\hline$X_{\mathrm{MeOS}}$ & $48 \%$ & $71 \%$ & $100 \%$ \\
\hline$A_{1}\left(10^{-20} \mathrm{~J}\right)$ & 0.0635 & 0.144 & 2.00 \\
\hline
\end{tabular}

To gain insight into the range of the dispersion forces in the films, we use the values of $A_{1}$ found above to estimate the interaction potential, $G\left(h_{0}\right)$, of our films. The results are displayed in Figure 5a. As one can see, the range of the $G\left(h_{0}\right)$ increases with $X_{\mathrm{MeOS}}$ and can extend up to distances of $\sim 15-20 \mathrm{~nm}$ from the substrate surface. These findings are qualitatively consistent with the fit values of $l_{\mathrm{t}}$ in Table 2 . To estimate how the interaction force may vary with $X_{\mathrm{MeOS}}$ at a distance equal to the range of the interaction, we define in our model the range of interaction to be the thickness where $G\left(h_{0}\right)=1.4 \times 10^{-6} \mathrm{~J} \cdot \mathrm{m}^{-2}$. This choice makes the range of the $X_{\mathrm{MeOS}}=100 \%$ films to be $20 \mathrm{~nm}$, and so similar to the value of 21.6 $\mathrm{nm}$ reported in Table 2. By using this criterion, the range of the $X_{\mathrm{MeOS}}=71 \%$ and $48 \%$ films are found to be 13.6 and $11.2 \mathrm{~nm}$, respectively. From the plot of $-d G\left(h_{0}\right) / d h_{0}$ shown in Figure $5 b$, we can see that the vdW interaction force at the interaction range decreases with $X_{\mathrm{MeOS}}$. Physically, we interpret this decrease of $-d G\left(h_{0}\right) / d h_{0}$ with $X_{\mathrm{MeOS}}$ to be caused by the 
interaction range being larger for larger $X_{\mathrm{MeOS}}$, and the fact that $d G\left(h_{0}\right) / d h_{0}$ generally decreases rapidly with $h_{0}$.

\section{REFERENCES}

1. Zhao, H.; Wang, Y. J.; Tsui, O. K. C. Dewetting induced by complete versus nonretarded van der Waals forces. Langmuir 2005, 21, 5817-5824.

2. Israelachvili, J., Intermolecular and Surface Forces. Academic Press: London, UK, 1992.

3. Koga, T.; Jiang, N.; Gin, P.; Endoh, M. K.; Narayanan, S.; Lurio, L. B.; Sinha, S. K. Impact of an Irreversibly Adsorbed Layer on Local Viscosity of Nanoconfined Polymer Melts. Phys. Rev. Lett. 2011, 107, 225901.

4. Sun, S.; Xu, H.; Han, J.; Zhu, Y.; Zuo, B.; Wang, X.; Zhang, W. The architecture of the adsorbed layer at the substrate interface determines the glass transition of supported ultrathin polystyrene films. Soft Matter 2016, 12, 8348-8358.

5. Hunter, C. A.; Lawson, K. R.; Perkins, J.; Urch, C. J. Aromatic interactions. J. Chem. Soc., Perkin Trans. 1 2001, 1, 651-669. 